Supporting information

\title{
Bifunctional $\mathrm{NbS}_{2}$-Based Asymmetric
}

\section{Heterostructure for Lateral and Vertical Electronic}

\section{Devices}

Bolun Wang ${ }^{\dagger}$, Hao Luo ${ }^{\dagger}$, Xuewen Wang $g^{\dagger}$ Enze Wang ${ }^{\dagger}$, Yufei Sun ${ }^{\dagger}, Y u$-Chien Tsai ${ }^{\ddagger}$, Hui Zhu ${ }^{\S}$, Peng Liu $u^{\ddagger}$ Kaili Jiang ${ }^{\ddagger}$ Kai Liu ${ }^{* \dagger}$

'State Key Laboratory of New Ceramics and Fine Processing, School of Materials Science and Engineering, Tsinghua University, Beijing 100084, China

Department of Physics and Tsinghua-Foxconn Nanotechnology Research Center, Tsinghua University, Beijing 100084, China

${ }^{\S}$ School of Microelectronics, Faculty of Information Technology, Beijing University of Technology, Beijing 100124, China

*E-mail: $\underline{\text { liuk@tsinghua.edu.cn }}$ 


\section{Optical and AFM characterization of $\mathrm{NbS}_{2}$}

a

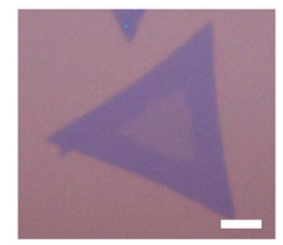

b

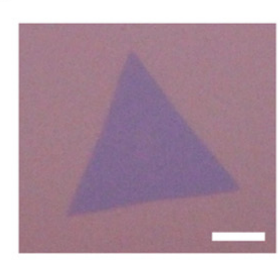

e

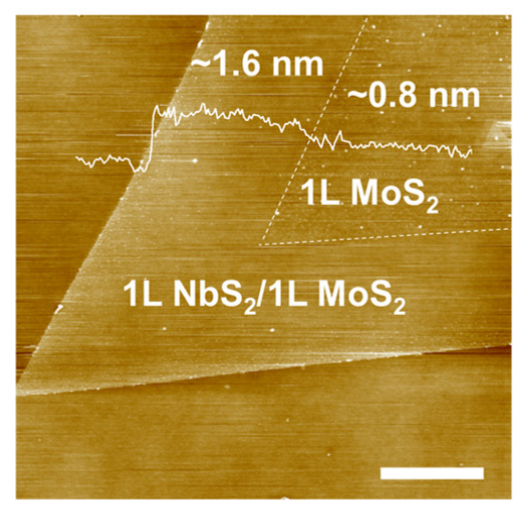

c

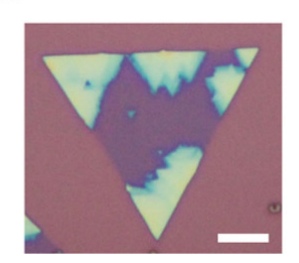

f

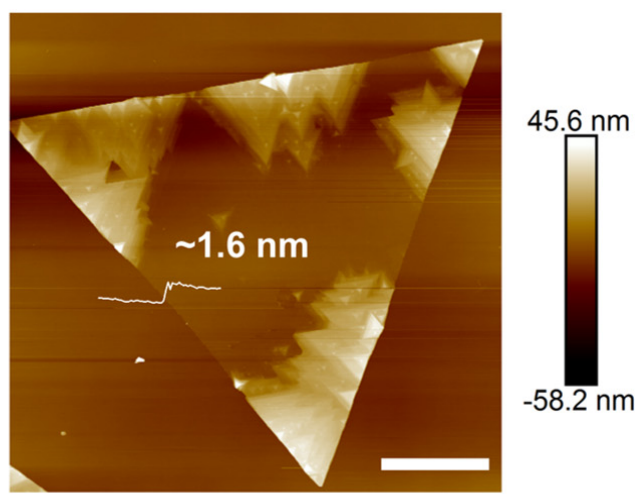

Figure S1. Optical and AFM characterization of $\mathrm{NbS}_{2}$. a-d) Optical images of different growth stages of $\mathrm{NbS}_{2}$ on $\mathrm{MoS}_{2}$ monolayers. Scale bar, $5 \mu \mathrm{m}$. e,f) AFM images of $\mathrm{NbS}_{2}$ corresponding to Figure S1a and Figure S1c. Scale bars, $3 \mu \mathrm{m}$ and $6 \mu \mathrm{m}$. 


\section{Optical images of $\mathrm{NbS}_{2}$ lateral devices}

a

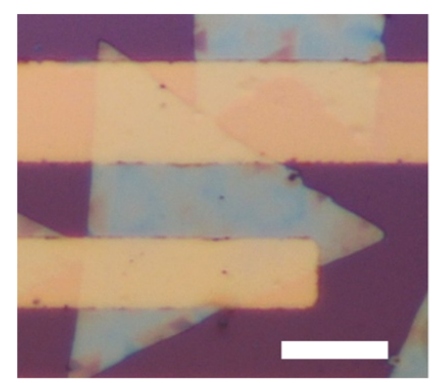

C

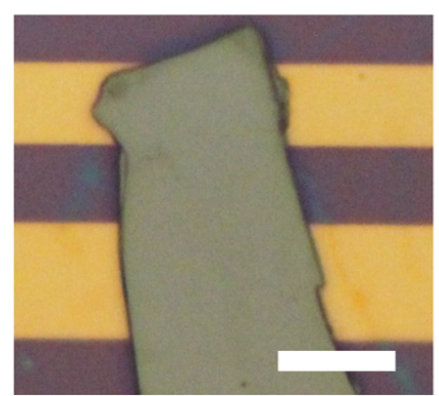

b

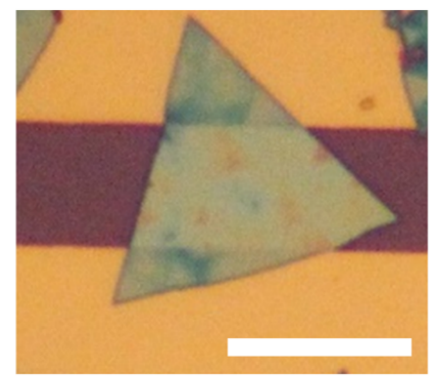

d

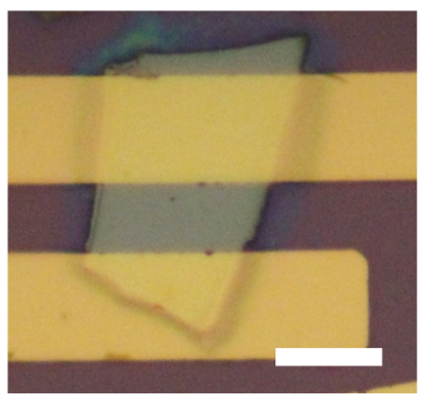

Figure S2. Optical images of $\mathrm{NbS}_{2}$ lateral devices. a) Optical images of Au electrodes on the memrisitive surface of $\mathrm{NbS}_{2}$. Scale bars, $10 \mu \mathrm{m}$. b) Optical images of the tunneling conductive surface of $\mathrm{NbS}_{2}$ on $\mathrm{Au}$ electrodes. Scale bars, $10 \mu \mathrm{m}$. c) Optical images of exfoliated $\mathrm{NbS}_{2}$ on $\mathrm{Au}$ electrodes. Scale bar, $10 \mu \mathrm{m}$. d) Optical images of Au electrodes on exfoliated $\mathrm{NbS}_{2}$. Scale bars, $10 \mu \mathrm{m}$. 


\section{I-V curves of exfoliated $\mathrm{NbS}_{2}$ lateral devices}

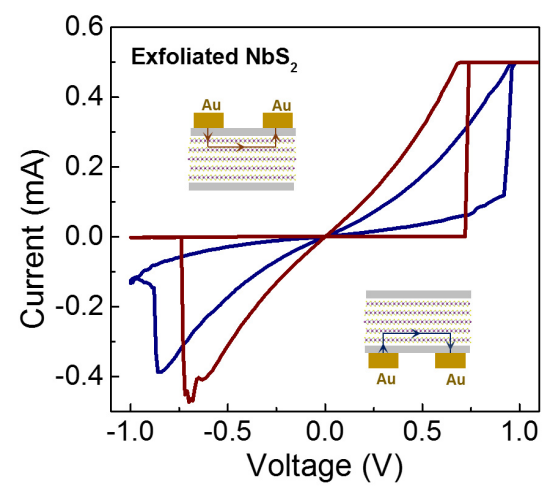

Figure S3. Typical current-voltage curves of the top surface and bottom surface of exfoliated $\mathrm{NbS}_{2}$. Top-left inset: Au top contacted device. Bottom-right inset: Au bottom contacted device.

\section{Crystal structures of $\mathrm{NbS}_{2}$}

a

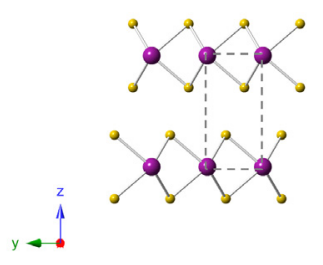

$2 \mathrm{H}$

b

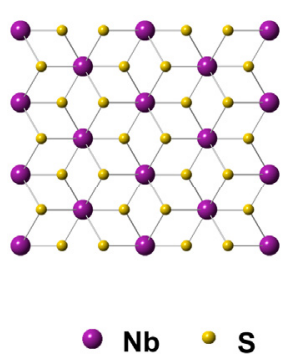

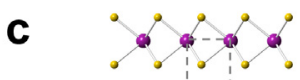

- go

?

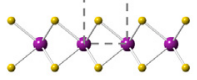

$3 R$

d

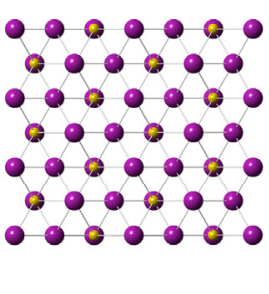

- Nb $\mathrm{N}$

Figure S4. Crystal structures of $\mathrm{NbS}_{2}$. a,b) Side view of the crystal structure of $2 \mathrm{H}-\mathrm{NbS}_{2}$ and $3 \mathrm{R}-\mathrm{NbS}_{2}$, respectively. c,d) Top view of the crystal structure of $2 \mathrm{H}-\mathrm{NbS}_{2}$ and $3 \mathrm{R}-\mathrm{NbS}_{2}$, respectively. 
5. Elemental analysis of the $\mathrm{NbS}_{2}$-based heterostructure

a

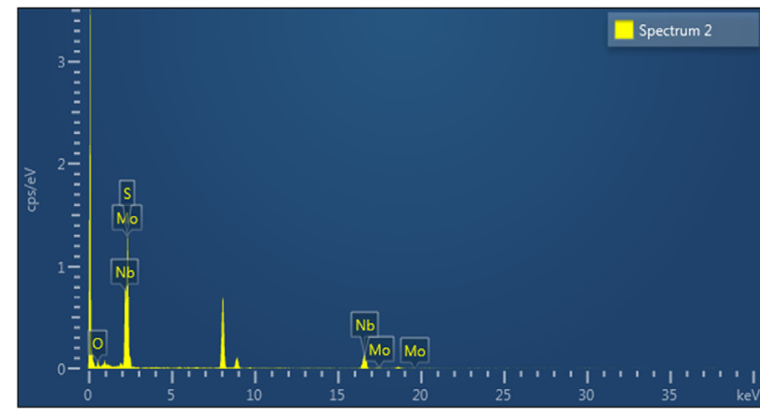

b

\begin{tabular}{|l|l|}
\hline Element & Atomic(\%) \\
\hline $\mathrm{O}$ & $\mathbf{9 . 4 3}$ \\
\hline $\mathrm{S}$ & 53.01 \\
\hline $\mathrm{Nb}$ & 34.99 \\
\hline Mo & 2.57 \\
\hline Total & 100 \\
\hline
\end{tabular}

Figure S5. Elemental analysis of the $\mathrm{NbS}_{2}$-based heterostructure. a) Elemental analysis of the transferred $\mathrm{NbS}_{2}$-based heterostructure on TEM girds. b) Element content of four element in the $\mathrm{NbS}_{2}$-based heterostructure.

\section{Raman spectra of the heterostructures exposed in air for different days}

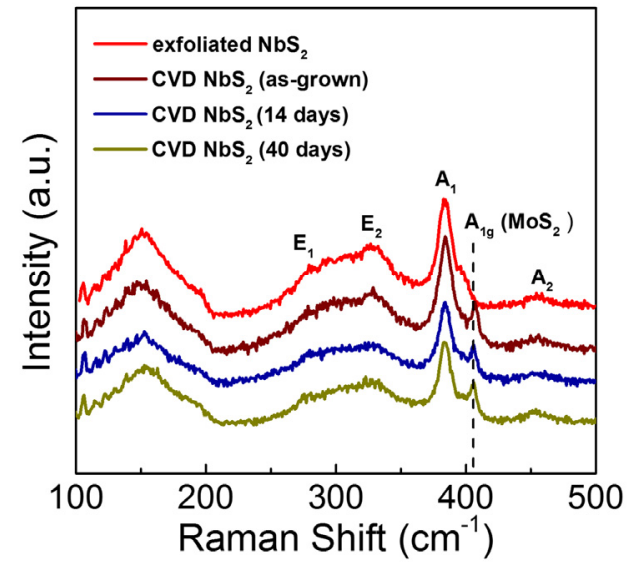

Figure S6. Raman spectra of the heterostructures exposed in air for different days. 
7. Optical images and performances of $\mathrm{NbS}_{2}$ contact $\mathrm{MoS}_{2}$ FET

a

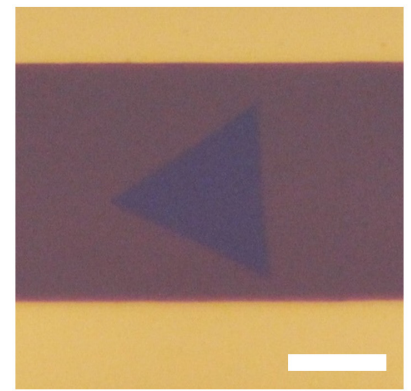

C

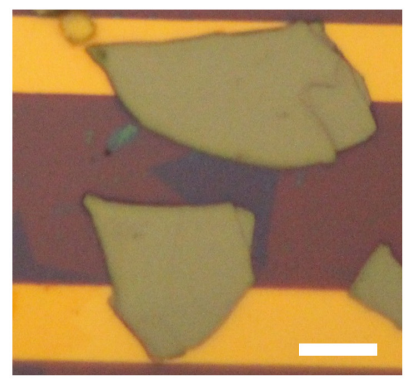

b

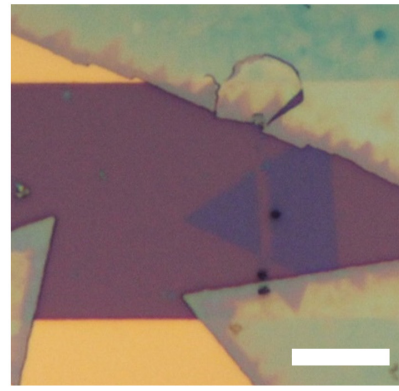

d

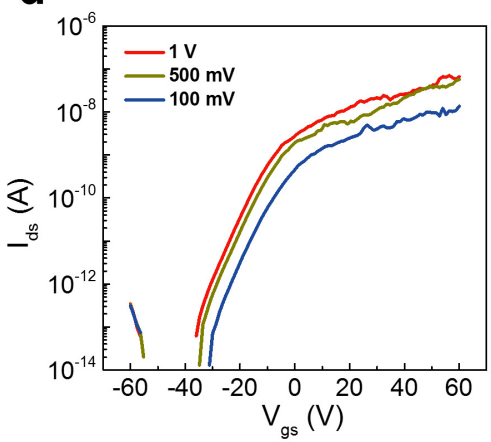

Figure S7. Optical images and performances of $\mathrm{NbS}_{2}$ contact $\mathrm{MoS}_{2}$ FET. a) Optical image of $\mathrm{MoS}_{2}$ transferred on $\mathrm{SiO}_{2} / \mathrm{Si}$ with pre-patterned $\mathrm{Au}$ electrodes. Scale bar, $10 \mu \mathrm{m}$. b) Optical image of $\mathrm{MoS}_{2}$ FET with the tunneling conductive surface of heterostructures contact, in which $\mathrm{MoS}_{2}$ triangle is cut into nearly rectangle shape to calculate the mobility. Scale bar, $\left.10 \mu \mathrm{m} . \mathrm{c}\right)$ Optical image of $\mathrm{MoS}_{2}$ FET with exfoliated $\mathrm{NbS}_{2}$ contact. Scale bar, $10 \mu \mathrm{m}$. d) $\mathrm{I}_{\mathrm{ds}}-\mathrm{V}_{\mathrm{gs}}$ curves of $\mathrm{MoS}_{2}$ FET with exfoliated $\mathrm{NbS}_{2}$ contact at different bias voltages. 
8. Schematic illustrations of the $\mathrm{MoS}_{2}$ FETs

a

Heterostructure contact

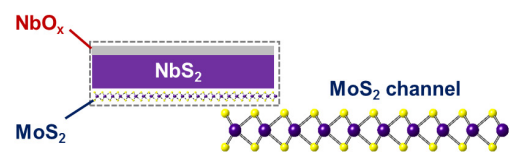

b

Exfoliated $\mathrm{NbS}_{2}$ contact

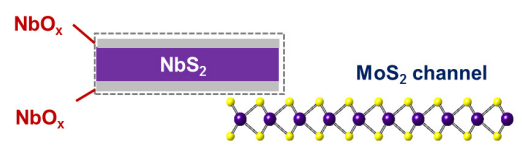

Figure S8. Schematic illustrations of the heterostructure-contacted $\mathrm{MoS}_{2}$ (a) and the exfoliated $\mathrm{NbS}_{2}$-contacted $\mathrm{MoS}_{2}(\mathrm{~b})$.

9. Resistance retention characteristic of the memristor based on the heterostructure

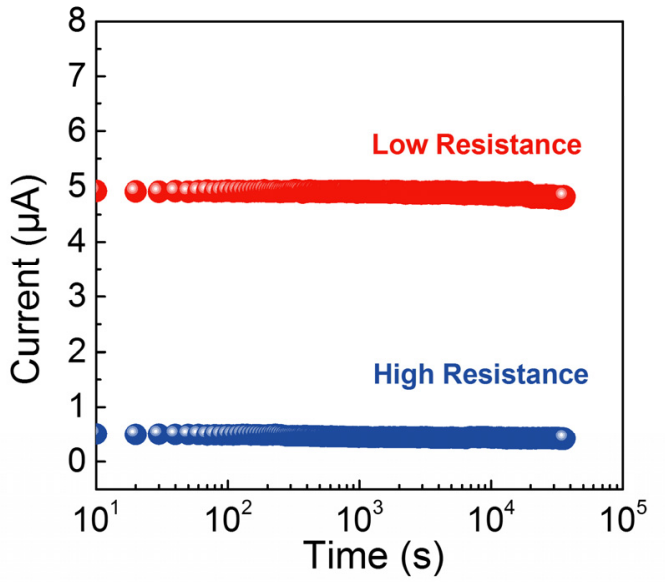

Figure S9. Retention characteristic of the memristor based on the heterostructure. The read voltage is $1 \mathrm{mV}$. 
10. Analysis of resistance switching mechanism of $\mathrm{NbS}_{2}$ memristors
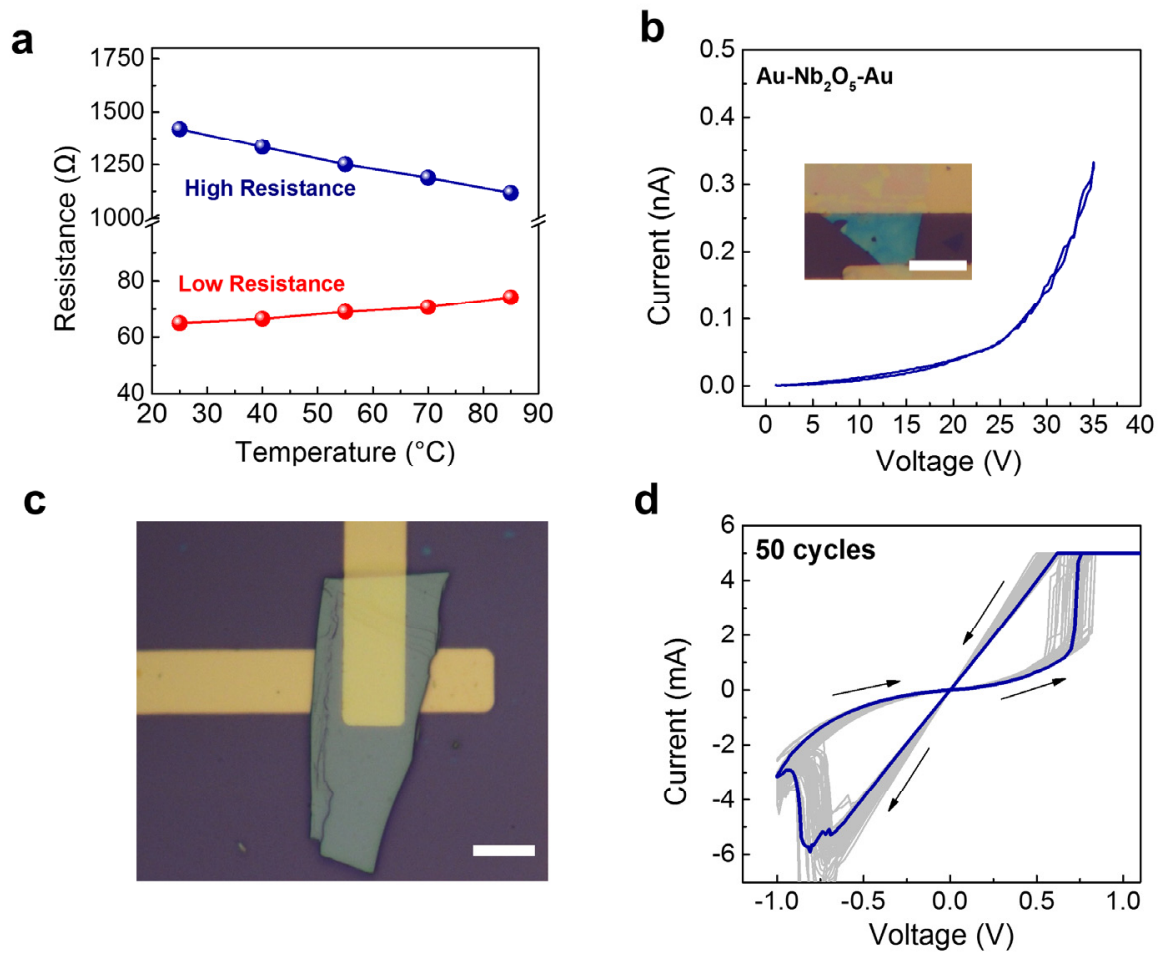

Figure S10. Analysis of resistance switching mechanism of $\mathrm{NbS}_{2}$ memristors. a) ResistanceTemperature curve of high resistance and low resistance of an $\mathrm{NbS}_{2}$ memristor. b) Current-

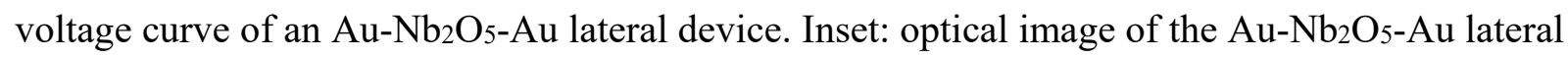
device. Scale bar, $10 \mu \mathrm{m}$. c) Optical image of an Au-exfoliated $\mathrm{NbS}_{2}$-Au vertical memristor. Scale bar, $10 \mu \mathrm{m}$. d) Current-voltage curves of an Au-exfoliated $\mathrm{NbS}_{2}$-Au vertical memristor. 
11. Schematic illustration of the resistance switching mechanism

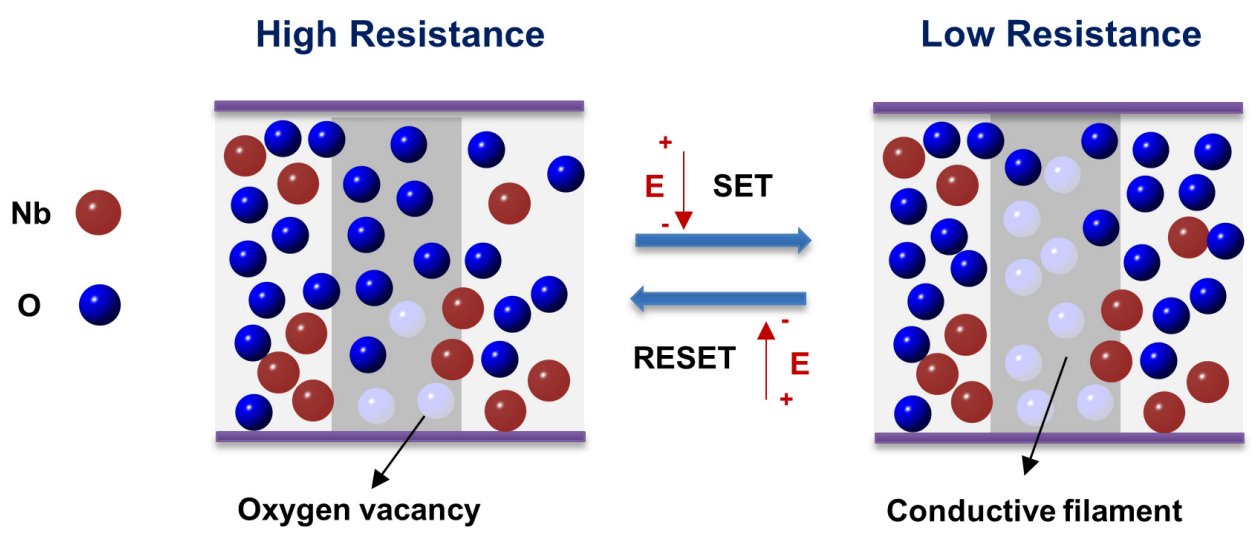

Figure S11. Schematic illustration of the resistance switching mechanism. Under high electric fields, metallic conductive filaments composed of oxygen vacancies can form in the semiconducting oxidation layer due to the oxygen ions migration, corresponding to the low resistance state. Then the high resistance state can be recovered by the reverse electric field owing to the rupture of conductive filaments.

\section{Comparison of current-voltage curves of a memristor tested in air and in vacuum}

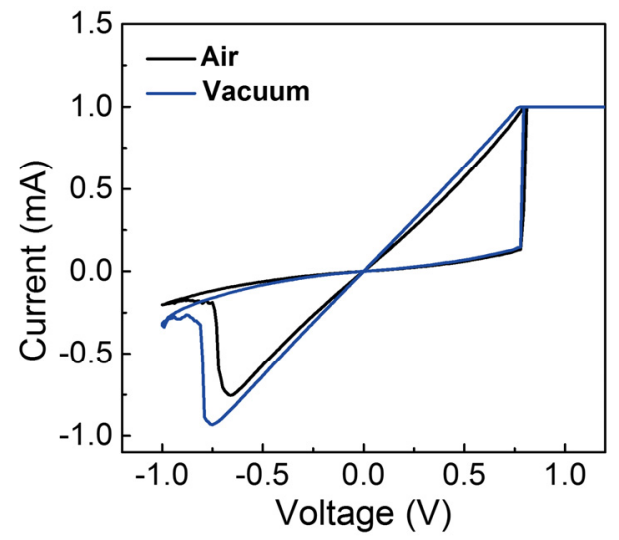

Figure S12. Comparison of current-voltage curves of a memristor tested in air and in vacuum. 
13. Performances of the memristors with different thicknesses of $\mathrm{NbS}_{2}$
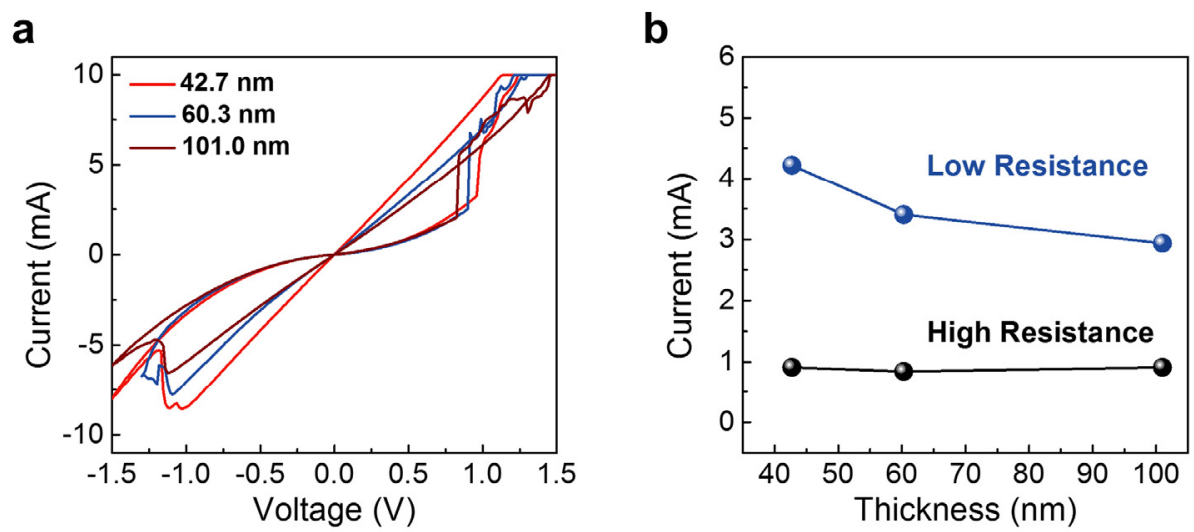

Figure S13. Performances of the memristors with different thicknesses of $\mathrm{NbS}_{2}$. a) Currentvoltage curves of memristors with different thicknesses of $\mathrm{NbS}_{2}$. b) Relationship of $\mathrm{NbS}_{2}$ thickness and the currents of memristors tested at $0.5 \mathrm{~V}$.

14. Performances of the memristors with different thicknesses of $\mathrm{NbO}_{\mathrm{x}}$

a

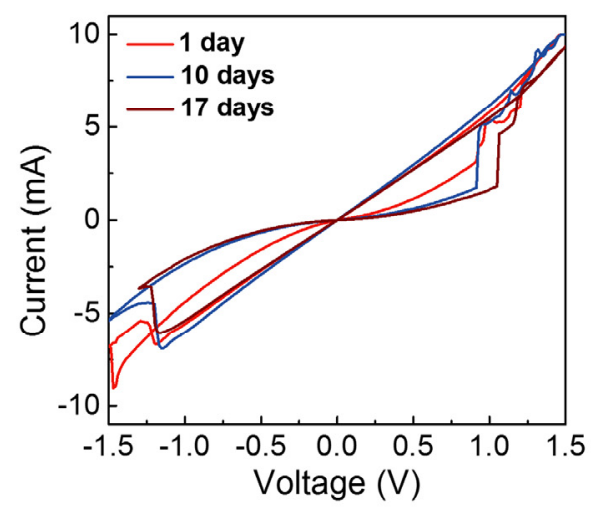

b

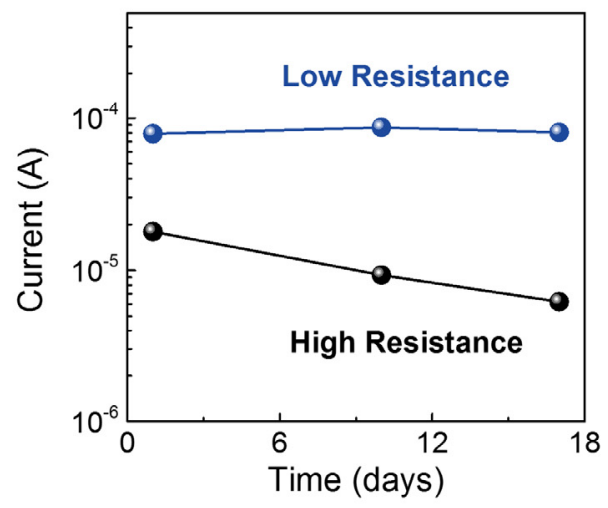

Figure S14. Performances of the memristors with different thicknesses of $\mathrm{NbO}_{\mathrm{x}}$. a) Currentvoltage curves of a memristor with different long periods of exposure in air. b) Dependence of the currents of the memristor at $0.15 \mathrm{~V}$ on the exposure time of the materials in air. 
15. Performance of a memristor based on the heterostructure oxidized at $300{ }^{\circ} \mathrm{C}$

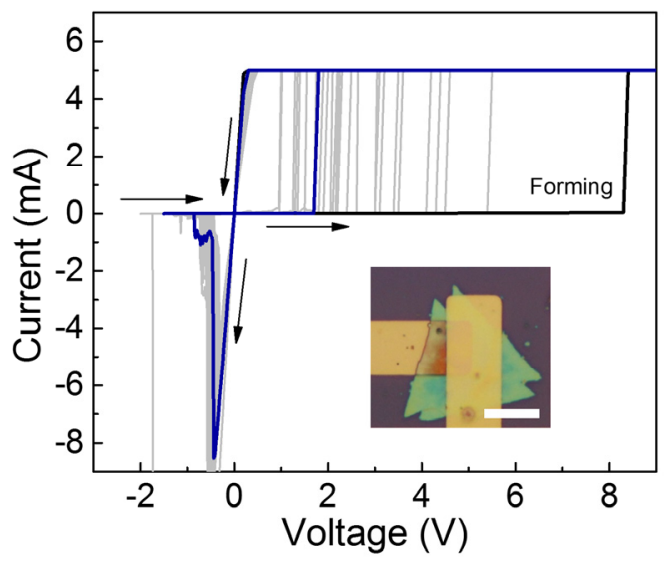

Figure S15. Current-voltage curves of a memristor based the heterostructure oxidized at $300{ }^{\circ} \mathrm{C}$ for $10 \mathrm{~min}$. Inset: optical image of the memristor. Scale bar, $10 \mu \mathrm{m}$.

\section{Repeated LTD and LTP tests}

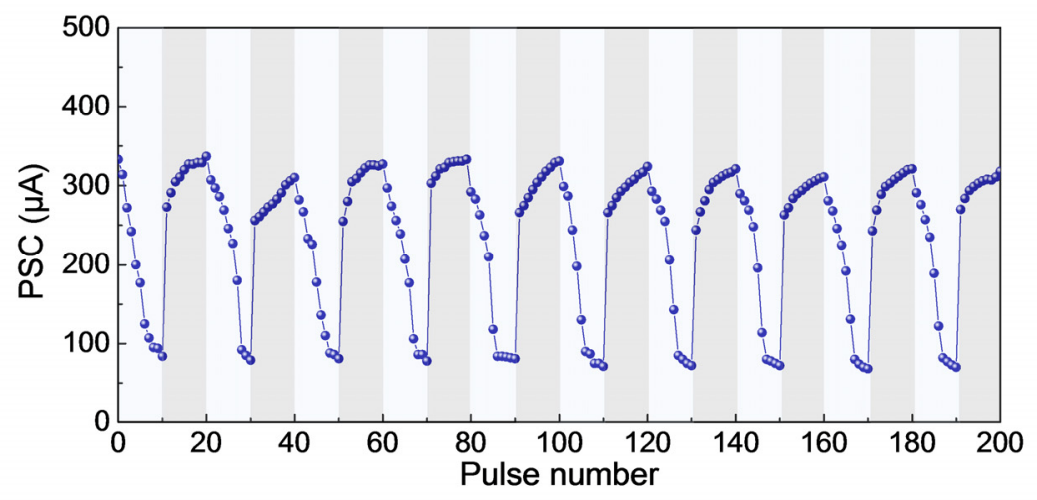

Figure S16. PSC of the memristor under ten cycles of pulses (10 consecutive negative pulses ($1.8 \mathrm{~V}, 10 \mu \mathrm{s})$ followed by 10 consecutive positive pulses $(1.8 \mathrm{~V}, 10 \mu \mathrm{s})$ for one cycle), which simulates repeated LTD and LTP properties of the synapse. The read voltage is $100 \mathrm{mV}$. 
17. Switching speed tests of a memristor

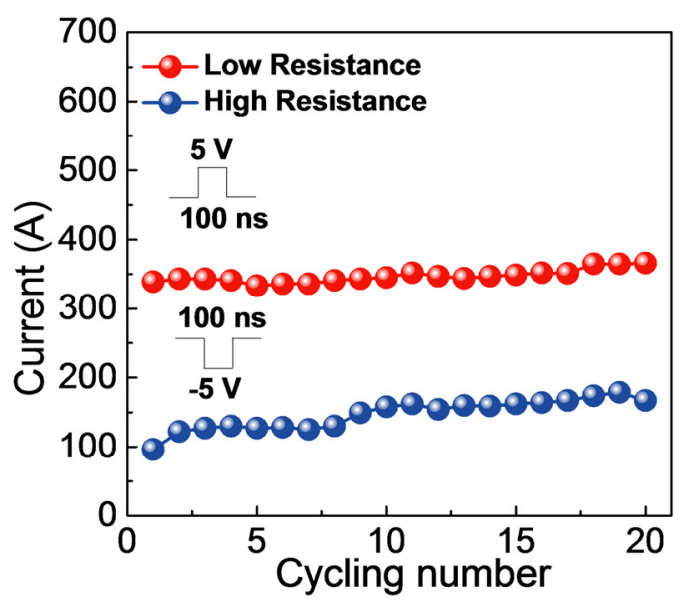

Figure S17. Resistance switching cycling under a series of positive and negative pulses. The read voltage is $100 \mathrm{mV}$.

\section{XPS tests of a transferred $\mathrm{NbS}_{2}$ on a PET substrate}

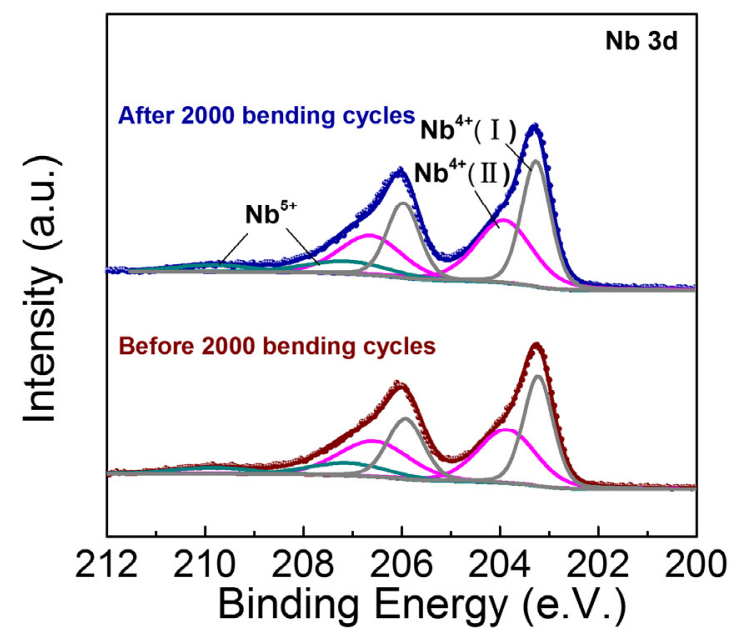

Figure S18. XPS binding energy of the $\mathrm{Nb}$ element of a transferred $\mathrm{NbS}_{2}$ on a PET substrate before and after 2000 bending cycles. 